\title{
Age-related murine hippocampal CA1 laminae oxidative stress measured in vivo by QUEnch-assiSTed (QUEST) MRI: impact of isoflurane anesthesia
}

\author{
Bruce A. Berkowitz • Robert H. Podolsky • Karen Lins Childers • Alexander Gow • \\ Brandy L. Schneider • Scott C. Lloyd • Kelly E. Bosse • Alana C. Conti • Robin Roberts • \\ Ali M. Berri • Emma Graffice • Kenan Sinan • Waleed Eliwat • Yimin Shen
}

Received: 12 November 2019/Accepted: 17 January 2020 /Published online: 25 January 2020

(C) The Author(s) 2020

\begin{abstract}
Age-related impairments in spatial learning and memory often precede non-familial neurodegenerative disease. Ex vivo studies suggest that physiologic age-related oxidative stress in hippocampus area CA1 may contribute to prodromal spatial disorientation and to morbidity. Yet, conventional blood or cerebrospinal fluid assays appear insufficient for early detection or management of oxidative stress within CA1 sub-regions in vivo. Here, we address this biomarker problem using a non-invasive MRI index of CA1 laminae oxidative stress based on reduction in R1 (= 1/T1) after anti-oxidant administration. An R1 reduction reflects quenching of continuous and excessive production of endogenous paramagnetic free radicals. Careful motion-correction image acquisi-
\end{abstract}

\footnotetext{
B. A. Berkowitz $(\bowtie) \cdot$ R. Roberts $\cdot$ A. M. Berri $\cdot$

E. Graffice $\cdot$ K. Sinan $\cdot$ W. Eliwat

Department of Ophthalmology, Visual and Anatomical Sciences,

Wayne State University School of Medicine, 540 E. Canfield,

Detroit, MI 48201, USA

e-mail: baberko@med.wayne.edu
}

\section{R. H. Podolsky $\cdot$ K. L. Childers}

Beaumont Research Institute, Beaumont Health, Royal Oak, MI 48073, USA

\section{A. Gow \\ Center for Molecular Medicine and Genetics, Wayne State University School of Medicine, Detroit, MI 48201, USA}

\footnotetext{
A. Gow

Department of Pediatrics, Wayne State University School of Medicine, Detroit, MI 48201, USA
}

tion, and avoiding repeated exposure to isoflurane, facilitates detection of hippocampus CA1 laminae oxidative stress with QUEnch-assiSTed (QUEST) MRI. Intriguingly, age- and isoflurane-related oxidative stress is localized to the stratum lacunosum of the CA1 region. Our data raise the possibility of using QUEST MRI and FDA-approved anti-oxidants to remediate spatial disorientation and later neurodegeneration with age in animals and humans.

Keywords Imaging $\cdot$ Free radicals $\cdot$ Reactive oxygen species $\cdot$ Brain

\footnotetext{
A. Gow

Department of Neurology, Wayne State University School of Medicine, Detroit, MI 48201, USA

B. L. Schneider - S. C. Lloyd - K. E. Bosse - A. C. Conti John D. Dingell VA Medical Center, Detroit, MI 48201, USA

B. L. Schneider - S. C. Lloyd - K. E. Bosse - A. C. Conti Deptarment of Neurosurgery, School of Medicine, Wayne State University School of Medicine, Detroit, MI 48201, USA

Y. Shen Department of Radiology, School of Medicine, Wayne State University School of Medicine, Detroit, MI 48201, USA
} 


\section{Introduction}

It is commonly suggested that hippocampal-dependent learning and memory are degraded in aged rodents and humans (Daugherty and Raz 2017; Kadish et al. 2009). Clinically meaningful disruptions in spatial learning and memory (e.g., a loss of goal location based on surrounding landmarks) are also associated with repeated anesthesia, such as isoflurane, a common experience for the older patient (Culley et al. 2003; Lin and Zuo 2011; Safavynia and Goldstein 2019). The spatial confusion in these apparently disparate conditions is commonly proposed to stem from oxidative stress within the mid- and posterior hippocampus (HC, human), or dorsal CA1 (CA1, rodents), which are specialized sub-regions of brain essential for encoding spatial information (Ali et al. 2006; Arimon et al. 2015; Chen et al. 2014; Fanelli et al. 2013; Forster et al. 1996; Frisoni et al. 2008; Hall et al. 2012; Han et al. 2015; Kanamaru et al. 2015; McManus et al. 2011; Moser et al. 1993; Mueller et al. 2007; Navarro et al. 2008; Nicolle et al. 2001; Pratico et al. 2001; Raz and Daugherty 2018; Strange et al. 2014; Tucsek et al. 2014). Importantly, prodromal administration of anti-oxidants preserves cognitive performance during healthy aging and in mice exposed to repeated isoflurane anesthesia (Carney et al. 1991; Clausen et al. 2010; Haxaire et al. 2012; Quick et al. 2008; Raghavendra and Kulkarni 2001; Shetty et al. 2014; Stoll et al. 1994; Wu et al. 2015; Zhang et al. 2018).

The benefits of anti-oxidant therapy, however, have not translated from preclinical studies to clinical practice because of the lack of assays for non-invasively evaluating local treatment efficacy (Raz et al. 2015). "Wet" biopsies are spatially non-specific and tissue biopsies are generally unavailable. Imaging brain oxidative stress and the effects of treatment in vivo has so far required the use of exogenous redox probes that are not FDA approved, limiting such studies to animal models (Bačić et al. 2015; Hall et al. 2012; Hou et al. 2018). The goal of this study is to begin to address the long-standing unmet need to measure oxidative stress in vivo with high spatial resolution using an endogenous biomarker to confirm localized efficacy of anti-oxidant treatment in hippocampus CA1 in both experimental models and, ultimately, in patients.

QUEnch-assiSTed (QUEST) MRI is a sensitive tool for non-invasive mapping of oxidative stress without an exogenous contrast agent (Berkowitz 2018; Berkowitz et al. 2019). The QUEST MRI oxidative stress index is a reduction in spin-lattice relaxation rate $\mathrm{R} 1(1 / \mathrm{T} 1)$ after acute anti-oxidant administration that maps the location of excessive production of paramagnetic free radicals (Berkowitz 2018). Most QUEST MRI studies to date have examined oxidative stress in photoreceptor neurons and find agreement with "gold standard" free radical measurements ex vivo when tested in several retinopathy models in mice anesthetized with urethane (Berkowitz 2018). However, our preliminary attempts to apply QUEST MRI to brains in adult mice repeatedly anesthetized with isoflurane were confounded by motion artifacts and other technical difficulties (see Discussion) (Berkowitz et al. 2017). It remains unclear if QUEST MRI can non-invasively detect age- or anesthesia-induced oxidative stress within hippocampus CA1 laminae in vivo.

In this study, we mitigate motion artifacts using imaging based on "periodically rotated overlapping parallel lines with enhanced reconstruction (PROPELLER)" (Berkowitz et al. 2017; Pipe 1999). Also, we test the sensitivity of QUEST MRI to measure hippocampal oxidative stress reported in aged mice, and young mice exposed to repeated isoflurane anesthesia (Carney et al. 1991; Clausen et al. 2010; Haxaire et al. 2012; Quick et al. 2008; Raghavendra and Kulkarni 2001; Shetty et al. 2014; Stoll et al. 1994; Zhang et al. 2018). With careful correction of motion and choice of anesthetic, our data demonstrate QUEST MRI is sensitive to oxidative stress in specific laminae within adult mouse CA1 hippocampus in vivo.

\section{Material and methods}

All animals were treated in accordance with the National Institutes of Health Guide for the Care and Use of Laboratory Animals, the Association for Research in Vision and Ophthalmology Statement for the Use of Animals in Ophthalmic and Vision Research, and Institutional Animal and Care Use Committee authorization. Animals were housed and maintained in 12-h:12-h light-dark cycle laboratory lighting, unless otherwise noted, and supplied with standard rodent chow and tap water ad libitum.

Animal groups First, we compared mouse brain R1 maps generated from either typical non-PROPELLER (i.e., Cartesian, $n=3)$ or PROPELLER $(n=3)$ 
acquisition sequences (not shown in Table 1); these mice were anesthetized with isoflurane during MRI examination. Second, for the repeated isoflurane anesthesia studies, 2-month male C57BL/6 (B6J) mice were bred inhouse from Jackson Laboratories (Bar Harbor, ME) breeders (Table 1). Repeated exposure of isoflurane, a complex I inhibitor, can have prolonged effects on brain tissue including production of hippocampus oxidative stress (Bajwa et al. 2018; Li et al. 2018; Ludwig et al. 2004; Wu et al. 2015; Zhang et al. 2018; Zimin et al. 2018). For this condition, mice were exposed to $5 \%$ isoflurane to induce anesthesia, monitored by loss of reactivity to the pedal withdrawal reflex, followed by maintenance of anesthesia with $2.5 \%$ isoflurane, delivered through a nose cone, for $15 \mathrm{~min}$. This isoflurane level is in line with that typically used to prepare rodents for further surgical procedures and was approved by veterinary staff at Wayne State University (Lowing et al. 2014). Animals were placed on a heating pad maintained at $37^{\circ} \mathrm{C}$ during anesthesia procedures and recovery. Following recovery, a subset of mice were subjected to isoflurane exposure again during MRI examination 6 days later for baseline image acquisition and at $24 \mathrm{~h}$ later following anti-oxidant exposure as described in the section below. In a companion study, mice were first anesthetized with isoflurane as above, but with urethane anesthesia during MRI examination. Third, in an aging model of hippocampus CA1 oxidative stress, we compared 2-month and 24-month male B6J mice derived from stocks at Jackson Laboratories, but bred and raised at the National Institute of Aging (B6NIA); NIA policy is to rederive mice from pedigreed stock every 6-7 years (https://www.nia.nih. gov/research/dab/animal-replacement-policy/colony- monitoring-and-history). These mice were anesthetized with urethane during MRI examination.

MRI The general mouse preparation for 2D MRI is well established in our laboratory (Berkowitz 2018). QUEST MRI following urethane anesthesia accurately assesses retinal oxidative stress in several mouse models (Berkowitz 2018). However, urethane is a terminal anesthetic and only cross-sectional studies are possible. This subgroup had two separate arms. One arm received $1 \mathrm{ml}$ of saline intraperitoneally (IP) at two time points (saline $\times 2$ ): $\sim 24 \mathrm{~h}$ and $\sim 1 \mathrm{~h}$ before acquisition of a T1 data set. The other arm received $1 \mathrm{mg} / \mathrm{kg}$ methylene blue (MB, IP, dissolved in saline). MB is an alternate electron transporter that effectively suppresses superoxide generation from mitochondria and various oxidases (Wen et al. 2011). The following day, approximately $1 \mathrm{~h}$ before the T1 MRI data collection, MB-treated mice received $50 \mathrm{mg} / \mathrm{kg} \alpha$-lipoic acid (ALA, IP, dissolved in saline and adjusted to $\mathrm{pH} \sim 7.4$ ); images were collected from a single slice ( -2 Bregma). ALA is a potent free radical neutralizer (Berkowitz et al. 2015; Berkowitz et al. 2016b; Gomes and Negrato 2014). Unless otherwise indicated, mice were anesthetized with urethane (36\% solution intraperitoneally; $0.083 \mathrm{ml} / 20 \mathrm{~g}$ animal weight, prepared fresh daily; Sigma-Aldrich, St. Louis, $\mathrm{MO})$ prior to MRI.

A different set of mice received isoflurane prior to MRI and isoflurane during MRI acquisition to monitor signal changes pre- (baseline) and post-anti-oxidant in the same mouse. Mice were anesthetized with isoflurane (3\% induction, 1.2\% maintenance) and a baseline $\mathrm{T} 1$ data set (as above). Mice were then given either saline or MB (vide supra) $30 \mathrm{~min}$ after recovery. The following

Table 1 Experimental conditions

\begin{tabular}{|c|c|c|c|c|c|}
\hline \multirow[t]{2}{*}{ Groups } & \multirow[t]{2}{*}{ Anesthetic during QUEST MRI } & \multicolumn{2}{|c|}{ Same mouse $(n)$} & \multicolumn{2}{|c|}{ Different mice $(n)$} \\
\hline & & Baseline & MB/ALA & Saline $\times 2$ & MB/ALA \\
\hline \multicolumn{6}{|l|}{ From Jackson Labs } \\
\hline 2 months C57BL/6 (B6J) & Urethane & & & 5 & 5 \\
\hline 2 months B6J, isoflurane (15 min, 1 week prior) & Urethane & & & 9 & 10 \\
\hline 2 months B6J isoflurane (15 min, 1 week prior & Isoflurane & 5 & & & \\
\hline \multicolumn{6}{|l|}{ From NIA } \\
\hline 2 months B6NIA & Urethane & & & 7 & 7 \\
\hline 2 months B6NIA & Urethane & & & 6 & 6 \\
\hline
\end{tabular}


day, each mouse given either saline or ALA (vide supra) was re-anesthetized with isoflurane, and another T1 data set obtained of the same brain slice as the previous day. In all cases, mice were humanely sacrificed at the end of the study. All experimental conditions and numbers of mice are summarized in Table 1.

In all studies, identical MRI sequences were used, and mice body temperature was regulated by a water bath that is integrated into the MRI cradle. T1 2D (collected using a PROPELLER sequence called BLADE (proprietary code) on the scanner used in this study with motion reconstruction) and T2 (multi-slice, collected with Cartesian acquisition) data sets were acquired on a $7 \mathrm{~T}$ scanner (Bruker ClinScan) using a receive only 4 -element phased array coil. Sixteen T2weighted images were acquired using a turbo spin echo sequence (repetition times (TRs) $2.05 \mathrm{~s}$; echo time (TE) $12 \mathrm{~ms}$; echo train length 7; number of averages 4; spacing between slices $0.4 \mathrm{~mm}$; slice thickness $400 \mu \mathrm{m} ; 12 \times 12 \mathrm{~mm}^{2}$, matrix size $192 \times 192$, in-plane resolution $62.5 \mu \mathrm{m}$ ). To measure $\mathrm{T} 1$, images with different repetition times (TRs) were acquired in the following order (number of averages in parentheses): TR $0.15 \mathrm{~s}(6), 3.50 \mathrm{~s}(1), 1.00 \mathrm{~s}(2), 1.90 \mathrm{~s}(1), 0.35 \mathrm{~s}(4)$, $2.70 \mathrm{~s}(1), 0.25 \mathrm{~s}(5)$, and $0.50 \mathrm{~s}$ (3). To compensate for reduced signal-to-noise ratios at shorter TRs, progressively more images were collected as the TR decreased. A spin-echo image was collected in a single transverse slice (echo time $(\mathrm{TE})=55 \mathrm{~ms}, 12 \times 12 \mathrm{~mm}^{2}$, matrix size $192 \times 192$, in-plane resolution $62.5 \mu \mathrm{m}$, slice thickness $400 \mu \mathrm{m}$, turbo factor 9) at each TR; please note that susceptibility changes caused by inhomogeneity's of the static magnetic field are expected to be nearly eliminated when using a spin-echo acquisition and thus unlikely to contribute to the observed signal. This saturation recovery approach provides precise $1 / \mathrm{T} 1$ values over a large range of signal-to-noise conditions and is routinely performed in our laboratory (Berkowitz 2018; Berkowitz et al. 2016a; Décorps et al. 1985; Freeman and Hill 1971; Haacke et al. 1999; Hsu et al. 2009).

MRI data analysis Because different hippocampus layers show different contrasts on T1- and T2weighted images (see, for example, "ImageBoost" in https://scalablebrainatlas.incf.org/mouse/WHS12), comparisons between these two data sets were useful for anatomical identification. Within each T1 data set of 23 slices, images acquired with the same TR were first registered (rigid body) and averaged to generate a stack of 8 images. The averaged images were registered across TRs. Thereafter, 1/T1 maps were calculated by fitting the data to a three-parameter T1 equation:

$\mathrm{y}=a+b^{*}\left(\exp \left(-c^{*} \mathrm{TR}\right)\right)$

where $a, b$, and $c$ are fitted parameters on a pixel-bypixel basis using R (v.2.9.0) and in-house scripts. We previously reported that day-to-day variations in $\mathrm{R} 1 \mathrm{can}$ be mitigated by removing slice bias in the $2 \mathrm{D}$ data and low signal-to-noise ratio, because the $\mathrm{T} 1$ estimate is highly dependent on the signal intensity of the TR 150-ms image and, thus, is imprecise (Chapter 18 in Haacke et al. 1999). By normalizing to the shorter TR, some of the bias can be removed and a more accurate $\mathrm{T} 1$ estimate obtained between days. We normalized within and between groups for signal intensity differences by first applying $3 \times 3$ Gaussian smoothing (performed three times) only to the TR 150 -ms image to suppress noise and emphasize signal. The smoothed image was then divided into the rest of the images of the T1 data set (Berkowitz 2018). An in-house R script was used to convert these 7 images into an R1 map.

To analyze the data, first the T1 and same slice T2 images were re-sized to $1440 \times 1440$ pixels using an ImageJ bilinear interpolation routine to iteratively draw a 5-pixel width segmented line region-of-interest (ROI) on the stratum lacunosum and stratum pyramidale layers as shown in Fig. 1 (Schneider et al. 2012). The R1 map was also re-sized to $1440 \times 1440$ pixels, but without any interpolation and ROIs applied to extract R1 values from each laminae (Fig. 1). This procedure was performed in triplicate for each ROI per mouse. These three values were used in the linear-mixed model analysis described below.

Statistical analysis Data are presented as mean \pm SEM. We conducted two separate analyses comparing the different groups of mice in Table 1: (1) a comparison of urethane-anesthetized mice exposed to isoflurane a week before QUEST MRI, and mice exposed to isoflurane a week before QUEST MRI in isoflurane-anesthetized mice; and (2) a comparison of 2-month B6NIA, 24-month B6NIA, and 2-month B6J urethane-anesthetized mice. All data contain repeated measures for each mouse, with at least triplicated and separate measurements for left and right sides of the brain. Further, mice repeatedly exposed to isoflurane were measured before and after being given 


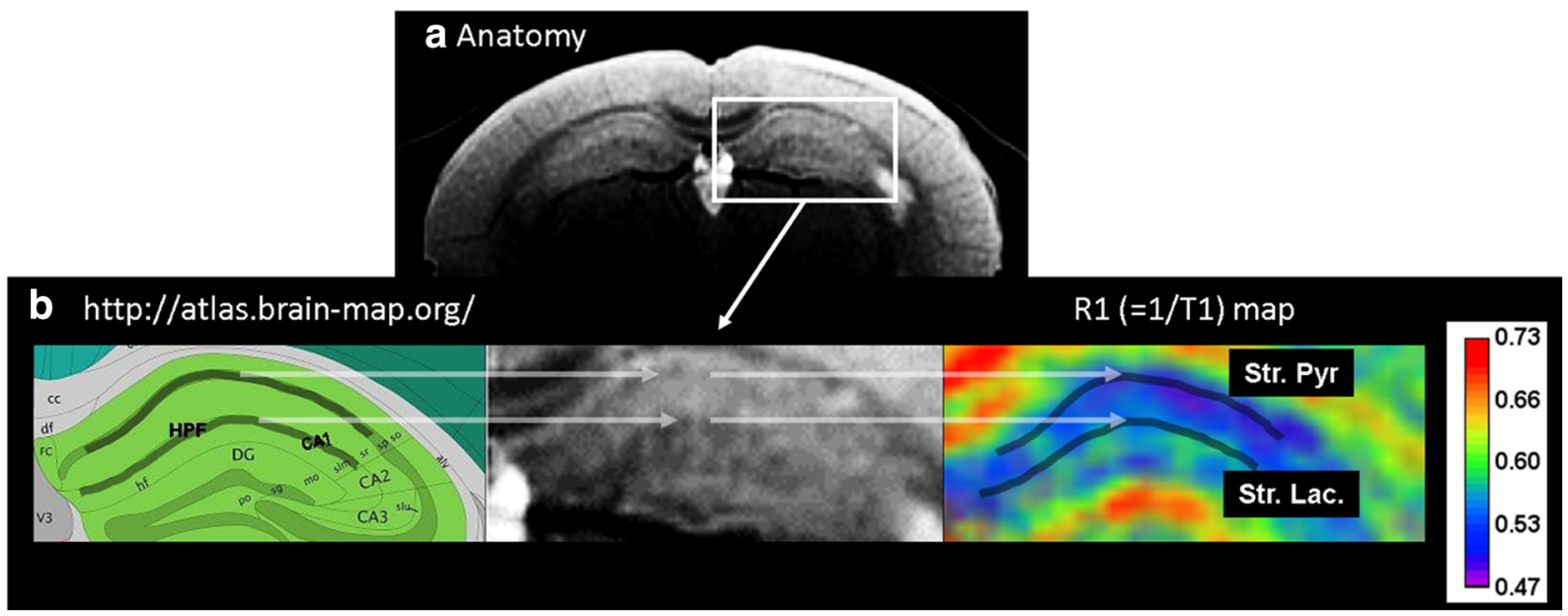

Fig. 1 a An MRI image illustrates the position of the slice in this study and ROI over the right hippocampus formation (HPF, white box). b Identification with the hippocampus CA1 stratum

anti-oxidants. With multiple measurements per mouse, we used linear-mixed models (PROC MIXED and PROC GLIMMIX in SAS) to analyze the data, with each specific model defined by the specific comparisons above. For all analyses, we first evaluated whether any random coefficients other than the random intercept had a large impact on model fit based on the Akaike and Schwarz Bayesian information criteria (AIC and BIC). We also evaluated heterogeneity in the residual variance and in the random effects among groups using AIC and BIC. More complex models were only favored when both AIC and BIC showed a reduction of at least 10. All fixed effects were evaluated using the likelihood ratio test. A significance level of 0.05 was used for tests of main effects, while interactions were tested using a significance level of 0.10 due to these tests having less power. All non-significant interactions were removed except for the group by antioxidant interaction to obtain the final model.

The final model for the comparison of urethane- and isoflurane-anesthetized mice included random coefficients for intercept, side, anti-oxidant exposure, and the interaction of side and anti-oxidant for each mouse nested within anesthetic. This model also included the fixed effects of anesthetic, side, anti-oxidant exposure, and all interactions among these main effects prior to testing fixed effects.

To compare the age groups, we initially analyzed all data together, evaluating the random coefficient for side and heterogeneity in the residual variance among groups using the AIC and BIC. These fit statistics indicated a large improvement of fit by including heterogeneity in the residual variance as well as by including the random pyramidale (Str. Pyr.) and stratum lacunosum (Str. Lac.) as gray lines drawn on the cartoon at the far left. Calibration bar indicates the range of R1s shown

coefficient for side. However, the resulting model had a large gradient and missing standard errors for some variances. As such, we decided to analyze the two sides separately. The final model for the comparison of age groups included a random intercept for each mouse nested within age group and a separate residual variance for each group. This model also included the fixed effects of anti-oxidant exposure, age group, and the interaction between anti-oxidant and age.

\section{Results}

Motion artifacts R1 maps generated with a PROPELLER sequence are sufficient to correct motion artifacts compared with a standard acquisition sequence, as is evident in the representative images in Fig. 2. Anatomical boundaries are easier to identify in PROPELLER images, which are visibly free of gross and artifactual asymmetries seen in R1 maps compared with those acquired using the typical approach (Fig. 2). The precision obtained using PROPELLER is supported by the results in Figs. 2, 3, and 4 below.

Repeated isoflurane-evoked hippocampus CA1 oxidative stress in vivo As shown in Fig. 3, pre-exposure to isoflurane 1 week prior to QUEST MRI using urethane anesthetic does not show oxidative stress in either strata lacunosum (Fig. 3a) or pyramidale (Fig. 3b). In contrast, evidence for hippocampus CA1 oxidative stress is 


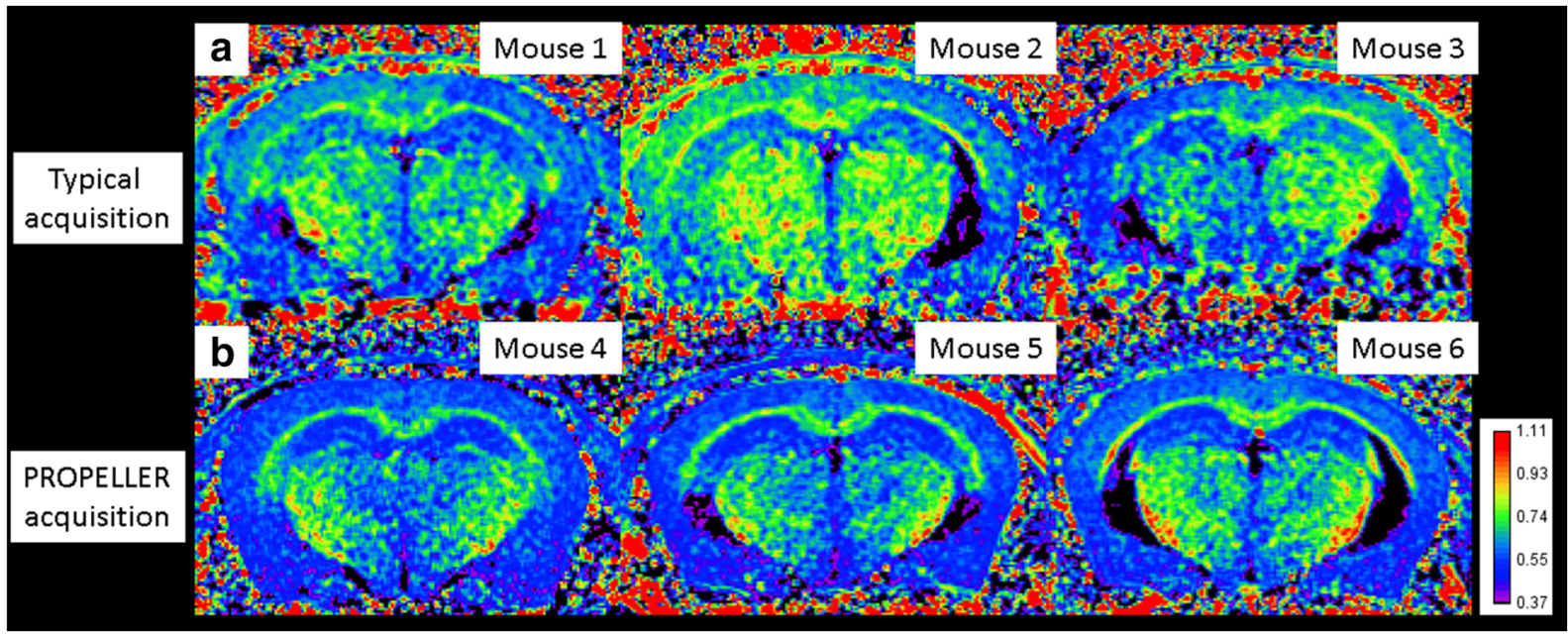

Fig. 2 Representative images showing R1 maps from data collected with a a typical acquisition sequence or $\mathbf{b}$ a periodically rotated overlapping parallel lines with enhanced reconstruction (PROPELLER) sequence (called BLADE on the system used in

suggested in animals pre-exposed to isoflurane 1 week prior to QUEST MRI examination performed using isoflurane (i.e., $\mathrm{R} 1$ is higher at baseline than in animals given urethane $(P=0.0205$; not shown on graph for clarity)) and confirmed by a reduction in R1 in a second this study). In all cases, post-processing normalization to the TR 150-ms image was performed to suppress B1 inhomogeneity artifacts, and coil and slice bias (Berkowitz et al. 2019). Calibration bar indicates the range of $\mathrm{R} 1 \mathrm{~s}$ shown

MRI study performed using isoflurane after giving antioxidants. In particular, CA1 oxidative stress is apparent in CA1 stratum lacunosum (Fig. 3). This result is statistically invariant between left and right hemispheres and the data are averaged for each mouse. We note that showing a

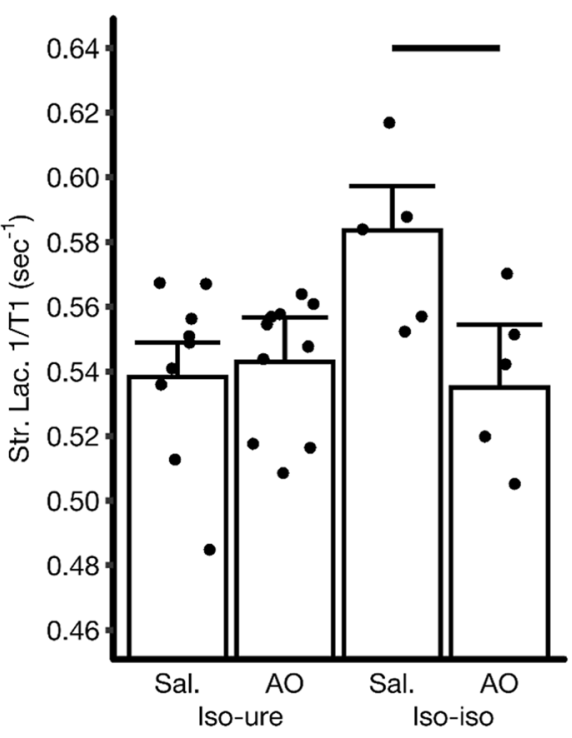

Fig. 3 Modeled R1 mean a stratum lacunosum (Str. Lac.) or b stratum pyramidale (Str. Pyr.) (illustrated in Fig. 2) for different B6J groups exposed to isoflurane a week before QUEST MRI in urethane-anesthetized mice (iso-ure, left two bars) vs. mice exposed to isoflurane a week before QUEST MRI in isofluraneanesthetized mice (iso-iso, right two bars). The number of animals in each group is presented in Table 1. Averages of R1 for each b

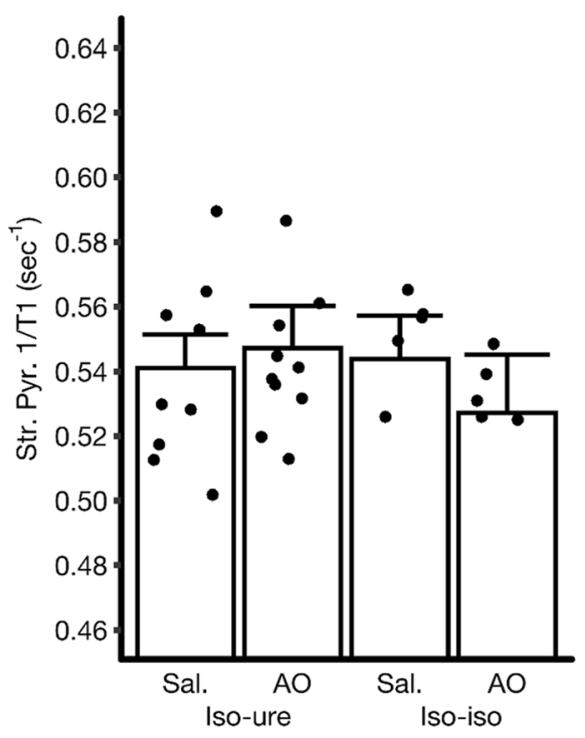

mouse are shown by circles. Error bars: SEM. Please note that the SEMs shown are based on a statistical modeling of the data. As such, the SEM is similar across groups. Reductions in R1 with anti-oxidants are considered to be an index of oxidative stress and significant changes are indicted with a horizontal black bar $(P<0.05)$ 

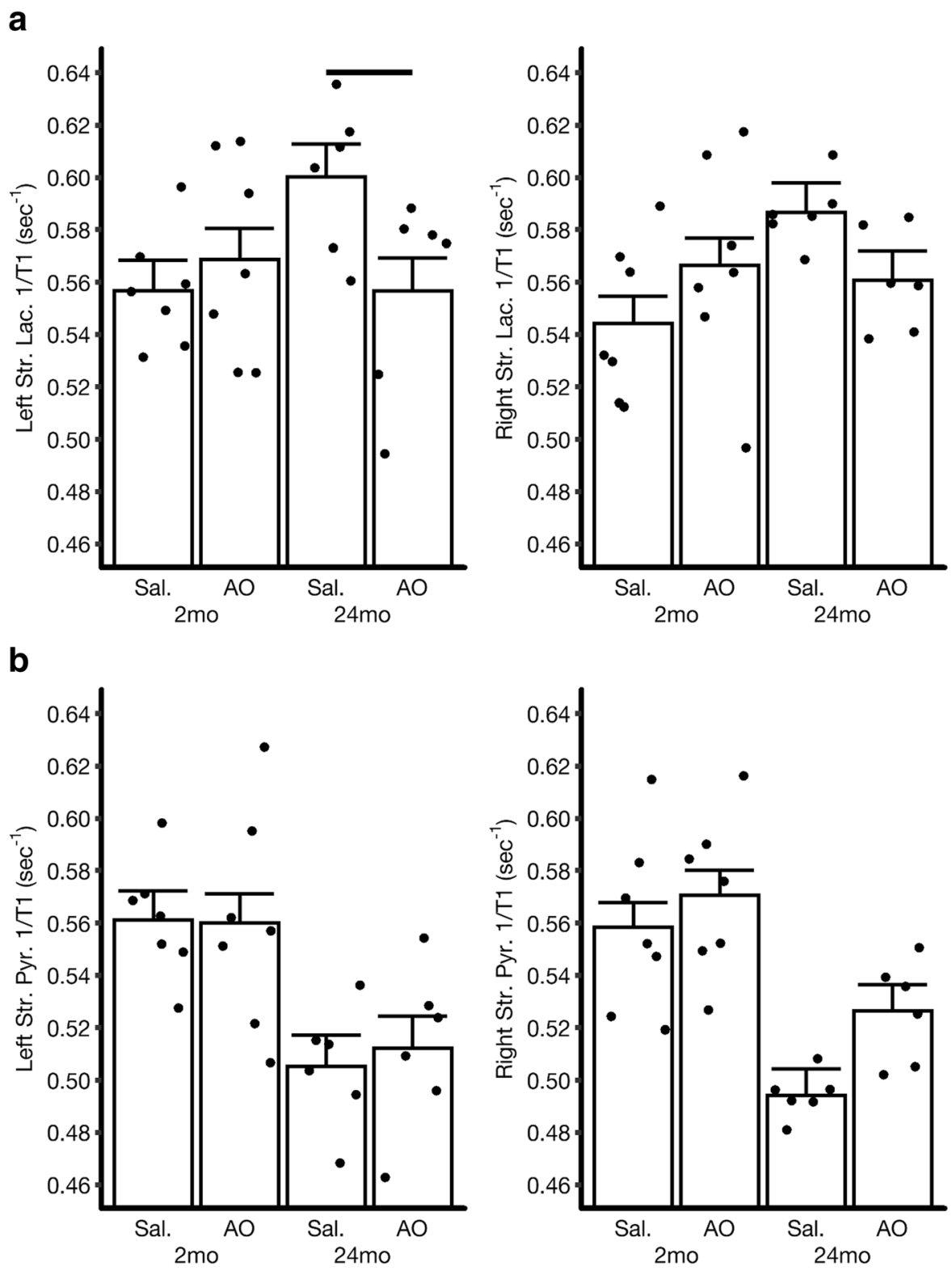

Fig. 4 Modeled R1 mean a left and right stratum lacunosum (Str. Lac.) or b stratum pyramidale (Str. Pyr.) (illustrated in Fig. 2) for different B6NIA groups aged either 2 months (left two bars) or 24 months (right two bars) from QUEST MRI scans of urethaneanesthetized mice. The number of animals in each group is presented in Table 1. Averages of R1 for each mouse are shown by

circles. Error bars: SEM. Please note that the SEMs shown are based on a statistical modeling of the data. As such, the SEM is similar across groups. Reductions in R1 with anti-oxidants are considered to be an index of oxidative stress and significant changes are indicted with a horizontal black bar $(P<0.05)$

both right and left comparisons would only be appropriate if we were showing the raw means and not those estimated from the final statistical model. This is because the modeled differences among the groups would be identical for both sides; adding these results would create a more complicated figure that does not contain new information.

Age-related CA1 oxidative stress in vivo Twenty-fourmonth B6NIA mice showed a suggestion of oxidative stress in CA1 stratum lacunosum since R1 is higher at baseline than in 24-mo B6NIA mice (left hemisphere, $P=0.0082$; right hemisphere, $P=0.0111$; not shown on graph for clarity). Oxidative stress was then established by a reduction in $\mathrm{R} 1$ after anti-oxidant as measured by 
QUEST MRI under urethane anesthesia (Fig. 4; see Discussion). Statistical significance is reached in the left stratum lacunosum but not the right, with the estimated effect of anti-oxidants on the right $(-0.026,95 \% \mathrm{CI}$ : $0.059,0.007)$ being about $1 / 2$ of that on the left $(-0.044$, $95 \%$ CI $-0.080,-0.007)$. In contrast, stratum pyramidale does not show oxidative stress in either hemisphere (i.e., no reduction in $\mathrm{R} 1$ with anti-oxidants). Also, no effect of vendor $(P>0.28)$ is found when comparing R1 values \pm anti-oxidants in 2 months B6J versus B6NIA mice (not shown).

\section{Discussion}

The goal of this study is to develop a viable QUEST MRI approach to measure layer-specific murine hippocampal CA1 oxidative stress in vivo. The present work focuses on addressing problems in our earlier report of QUEST MRI for measuring hippocampal formation oxidative stress (Berkowitz et al. 2017). The first problem is that substantial motion artifacts in the images of the hippocampal formation collected using a standard acquisition yield relatively few animals with usable data (Berkowitz et al. 2017). Here, we show that a PROPELLER acquisition sequence solved this problem in the adult mouse brain (Fig. 2) (Berkowitz et al. 2019). The second problem is a paradoxical finding of hippocampus CA1 oxidative stress in control mice repeatedly exposed to isoflurane. This problem was likely masked in our initial report by combining control groups and/or by the motion artifacts that are clearly evident in Fig. 2. In addition, isoflurane-evoked oxidative stress in the hippocampus of control mice can confound interpretation of QUEST MRI data in experimental models (Ni et al. 2017; Zhang et al. 2018; Zimin et al. 2018). Here, we find limiting isoflurane to a single pre-examination exposure followed by urethane anesthesia is useful for measuring the presence (in experimental groups) or absence (in controls) of oxidative stress in the murine brain in vivo (Fig. 3). These data support and extend the suitability of urethane for QUEST MRI first reported in experimental retina studies (Berkowitz 2018), a likely result of urethane's ability to minimally alter, for example, functional connectivity, and autonomic and cardiovascular systems (Hara and Harris 2002; Paasonen et al. 2018). A related problem was our prior use of a corpus callosum ROI near hippocampus CA1 (Berkowitz et al.
2017). A recent study identifies profound and prolonged changes in corpus callosum microstructure after isoflurane anesthesia in control mice - a clear confound in the interpretation of R1 (Bajwa et al. 2018; Berkowitz et al. 2017; Hirata et al. 2011; Li et al. 2018; Xu et al. 2018; Zhang et al. 2018; Zimin et al. 2018). Correcting motion artifacts and avoiding repeated isoflurane anesthesia increases the reliability of QUEST MRI as a method for measuring oxidative stress in murine hippocampus CA1 laminae in vivo.

To strengthen our statistical power, our a priori design was to examine only two hippocampus laminae for oxidative stress. These two layers were chosen based on studies showing that stratum lacunosum has relatively greater energy metabolism (e.g., glucose utilization, and succinate dehydrogenase and cytochrome c oxidase activity), and more prominent vascularity than stratum pyramidale (Borowsky and Collins 1989; Gulyas et al. 2006; Kann 2016; Kubota et al. 1993; Kugler et al. 1988; Mattiasson et al. 2003; Nicolle et al. 2001; Shimada et al. 1992; Shimada et al. 1994; Shimada et al. 1989; Wang et al. 2005; Wilde et al. 1997).

In contrast to the current study, several ex vivo studies have suggested that the stratum pyramidale is particularly susceptible to developing oxidative stress (Fekete et al. 2008; La et al. 2019; Santini et al. 2015; Stebbings et al. 2016). This apparent disparity with our findings may arise from substantial methodological differences, where the majority of the previous studies utilize dihydroethidium (DHE) staining as a marker for oxidative stress (Du et al. 2013; Michalski et al. 2014; Santini et al. 2015). Ex vivo staining with DHE reveals the cumulative effects of oxidative stress - that of oxidized DNA in the nucleus - even if superoxide is not produced in this organelle (Du et al. 2013; Michalski et al. 2014). Concerns about using DHE, and its derivatives like MitoSox, continue to be raised (Cheng et al. 2018; Xiao and Meierhofer 2019). Also, comparing DHE and QUEST MRI indices may not be justified because DHE measures oxidative damage that builds up over time, in contrast to QUEST MRI which is a snapshot of excessive free radical production. In support of this notion, QUEST MRI results show spatial agreement with data from ex vivo probes such as lucigenin or dihydrodichlorofluorescein (DCF), assays that provide snapshot measurements of free radical production in retinopathy models (Berkowitz 2018). However, we cannot rule out the possibility that oxidative stress was not detected in stratum pyramidale because of its small thickness leading to increased partial volume averaging. 
Because of the potentially confounding problems of variable post-mortem intervals for ex vivo assays of oxidative stress and/or damage (above), we have argued that QUEST MRI can be used as a stand-alone in vivo assay of oxidative stress (Berkowitz 2018; Cheng et al. 2018; Xiao and Meierhofer 2019). QUEST MRI results, as noted above, are in agreement with ex vivo lucigenin and DCF assays in retinopathy. Also, the detection of excessive free radical production by QUEST MRI has been confirmed in a phantom study of the xanthine-xanthine oxidase reaction (Berkowitz 2018). Further, the biophysics underlying QUEST MRI is consistent with the expected impact of a continuous and asynchronous production of paramagnetic free radicals on R1 (Berkowitz 2018). Furthermore, the results of the present study demonstrate agreement between QUEST MRI and the extant literature which establish that repeated isoflurane causes excessive production of free radicals in the hippocampus (Hirata et al. 2011; Li et al. 2018; Wu et al. 2015; Xu et al. 2018; Zhang et al. 2018; Zimin et al. 2018). Similarly, the present QUEST MRI data from the 24-month mice are consistent with reports of hippocampal oxidative stress during aging, which is commonly proposed to arise from reductions in anti-oxidant defense mechanisms over time (Ahn et al. 2016; Ali et al. 2006; Antier et al. 2004; Dugan et al. 2009; Fukui et al. 2002; Hall et al. 2012; Haxaire et al. 2012; Lacoste et al. 2017; Nicolle et al. 2001; Stebbings et al. 2016).

In our analyses of oxidative stress in the CA1 of aged mice, the most robust QUEST MRI evidence was found in the left CA1 stratum lacunosum of 24-month-old mice. We were not able to evaluate whether the antioxidant response differs between the two sides due to computational problems in fitting an appropriate statistical models when the two sides were combined. The actual anti-oxidant response in 24 month-old mice for the two sides may be similar, and intermediate to estimates obtained separately for the two sides. As such, our study does not provide evidence for age-related lateralization of hippocampus CA1 oxidative stress. Further, QUEST MRI measurements obtained before or after the 24-month time point may show a different spatial patterns with regard to hippocampus CA1 oxidative stress.

The results of this study, and those from the literature, highlight the need for caution when using isoflurane which, when used repeatedly, is not benign as is often assumed (Bajwa et al. 2018; Crystal et al. 2012; Huang et al. 2018; Li et al. 2018; Lin and Zuo 2011; Ni et al. 2017; Paasonen et al. 2018; Wu et al. 2015; Zhang et al. 2018).
Isoflurane is known to induce cerebral hyperemia and suppresses neuronal activity (Kehl et al. 2002; Pan et al. 2015; Schroeter et al. 2014; Toyama et al. 2004). More work is needed to investigate variables such as dose, and post-isoflurane duration, to explore how the first isoflurane exposure apparently primes the brain to produce hippocampus CA1 stratum lacunosum oxidative stress in vivo after second and third isoflurane exposures (Fig. 3).

In summary, we developed a QUEST MRI protocol that uniquely measures murine hippocampus CA1 laminae oxidative stress in vivo. This study addresses longstanding shortcomings of conventional assays in experimental studies. We speculate that applications of QUEST MRI in humans will be possible in the future. Since urethane is not clinically translatable, we anticipate that more work will be needed to identify acceptable anesthesia protocols in patients that require sedation for their MRI examination. QUEST MRI is based on an endogenous contrast mechanism and is performed with FDA-approved anti-oxidants and thus is a promising method to facilitate testing of anti-oxidant treatments for mitigating behavioral spatial disorientation, and perhaps other aspects of oxidative stress-associated cognitive dysfunction (Raz and Daugherty 2018).

Acknowledgments We thank Drs. Naftali Raz, David Bissig, Geoffrey Murphy, Olivier Thibault, and Brian Bennett for their comments and insight. This work was supported by resources and facilities at the John D. Dingell VA Medical Center (ACC).

Funding information This work was supported by grants to AG from the National Institutes of Health, National Institute of Neurological Disorders and Stroke (NS043783 and NS067157), and the National Multiple Sclerosis Society (RG4639 and RG4906); to BAB from National Institutes of Health, National Eye Institute (R01EY026584), and National Institute of Aging (R01AG058171); and the Richard Barber Interdisciplinary Research Program at Wayne State University (ACC and BAB).

\section{Compliance with ethical standards}

Conflict of interest The authors declare that they have no conflict of interest.

Open Access This article is licensed under a Creative Commons Attribution 4.0 International License, which permits use, sharing, adaptation, distribution and reproduction in any medium or format, as long as you give appropriate credit to the original author(s) and the source, provide a link to the Creative Commons licence, and indicate if changes were made. The images or other third party material in this article are included in the article's Creative Commons licence, unless indicated otherwise in a credit line to the material. If material is not included in the article's Creative 
Commons licence and your intended use is not permitted by statutory regulation or exceeds the permitted use, you will need to obtain permission directly from the copyright holder. To view a copy of this licence, visit http://creativecommons.org/licenses/by/ $4.0 \%$

\section{References}

Ahn JH, Chen BH, Shin BN, Lee TK, Cho JH, Kim IH, Park JH, Lee JC, Tae HJ, Lee CH, Won MH, Lee YL, Choi SY, Hong S (2016) Comparison of catalase immunoreactivity in the hippocampus between young, adult and aged mice and rats. Mol Med Rep 14(1):851-856

Ali SS, Xiong C, Lucero J, Behrens MM, Dugan LL, Quick KL (2006) Gender differences in free radical homeostasis during aging: shorter-lived female C57BL6 mice have increased oxidative stress. Aging Cell 5(6):565-574

Antier D, Carswell HV, Brosnan MJ, Hamilton CA, Macrae IM, Groves S, Jardine E, Reid JL, Dominiczak AE (2004) Increased levels of superoxide in brains from old female rats. Free Radic Res 38(2):177-183

Arimon M, Takeda S, Post KL, Svirsky S, Hyman BT, Berezovska O (2015) Oxidative stress and lipid peroxidation are upstream of amyloid pathology. Neurobiol Dis 84:109-119

Bačić G, Pavićević A, Peyrot F (2015) In vivo evaluation of different alterations of redox status by studying pharmacokinetics of nitroxides using magnetic resonance techniques. Redox Biol 8:226-242

Bajwa NM, Lee JB, Halavi S, Hartman RE, Obenaus A (2018) Repeated isoflurane in adult male mice leads to acute and persistent motor decrements with long-term modifications in corpus callosum microstructural integrity. J Neurosci Res

Berkowitz BA (2018) Oxidative stress measured in vivo without an exogenous contrast agent using QUEST MRI. J Mag Reson 291:94-100

Berkowitz BA, Bredell BX, Davis C, Samardzija M, Grimm C, Roberts R (2015) Measuring in vivo free radical production by the outer retina measuring retinal oxidative stress. Invest Ophthalmol Vis Sci 56(13):7931-7938

Berkowitz BA, Bissig D, Roberts R (2016a) MRI of rod cell compartment-specific function in disease and treatment inávivo. Prog Retin Eye Res 51:90-106

Berkowitz BA, Lewin AS, Biswal MR, Bredell BX, Davis C, Roberts R (2016b) MRI of retinal free radical production with laminar resolution in vivo free radical production with laminar resolution in vivo. Invest Ophthalmol Vis Sci 57(2):577-585

Berkowitz BA, Lenning J, Khetarpal N, Tran C, Wu JY, Berri AM, Dernay K, Haacke EM, Shafie-Khorassani F, Podolsky RH, Gant JC, Maimaiti S, Thibault O, Murphy GG, Bennett BM, Roberts R (2017) In vivo imaging of prodromal hippocampus CA1 subfield oxidative stress in models of Alzheimer disease and Angelman syndrome. FASEB J

Berkowitz BA, Romero R, Podolsky RH, Lins-Childers KM, Shen Y, Rosales T, Wadghiri YZ, Hoang DM, Arenas-Hernandez M, Garcia-Flores V, Schwenkel G, Panaitescu B, GomezLopez N (2019) QUEST MRI assessment of fetal brain oxidative stress in utero. Neuroimage 200:601-606
Borowsky IW, Collins RC (1989) Metabolic anatomy of brain: a comparison of regional capillary density, glucose metabolism, and enzyme activities. J Comp Neurol 288(3):401-413

Carney JM, Starke-Reed PE, Oliver CN, Landum RW, Cheng MS, Wu JF, Floyd RA (1991) Reversal of age-related increase in brain protein oxidation, decrease in enzyme activity, and loss in temporal and spatial memory by chronic administration of the spin-trapping compound N-tert-butyl-alphaphenylnitrone. Proc Natl Acad Sci U S A 88(9):3633-3636

Chen L, Na R, Ran Q (2014) Enhanced defense against mitochondrial hydrogen peroxide attenuates age-associated cognition decline. Neurobiol Aging 35(11):2552-2561

Cheng G, Zielonka M, Dranka B, Kumar SN, Myers CR, Bennett B, Garces AM, Dias Duarte Machado LG, Thiebaut D, Ouari O, Hardy M, Zielonka J, Kalyanaraman B (2018) Detection of mitochondria-generated reactive oxygen species in cells using multiple probes and methods: potentials, pitfalls, and the future. J Biol Chem 293(26):10363-10380

Clausen A, Doctrow S, Baudry M (2010) Prevention of cognitive deficits and brain oxidative stress with superoxide dismutase/ catalase mimetics in aged mice. Neurobiol Aging 31(3):425-433

Crystal GJ, Malik G, Yoon SH, Kim SJ (2012) Isoflurane late preconditioning against myocardial stunning is associated with enhanced antioxidant defenses. Acta Anaesthesiol Scand 56(1):39-47

Culley DJ, Baxter M, Yukhananov R, Crosby G (2003) The memory effects of general anesthesia persist for weeks in young and aged rats. Anesth Analg 96(4):1004-1009 table of contents

Décorps M, Laval M, Confort S, Chaillout JJ (1985) Signal to noise and spatial localiztion of NMR spectra with a surface coil and the saturation-recovery sequence. J Magn Reson 61:418-425

Du Y, Veenstra A, Palczewski K, Kern TS (2013) Photoreceptor cells are major contributors to diabetes-induced oxidative stress and local inflammation in the retina. Proc Natl Acad Sci 110(41):16586-16591

Dugan LL, Ali SS, Shekhtman G, Roberts AJ, Lucero J, Quick KL, Behrens MM (2009) IL-6 mediated degeneration of forebrain GABAergic interneurons and cognitive impairment in aged mice through activation of neuronal NADPH oxidase. PLoS One 4(5):e5518

Fanelli F, Sepe S, D'Amelio M, Bernardi C, Cristiano L, Cimini A, Cecconi F, Ceru' MP, Moreno S (2013) Age-dependent roles of peroxisomes in the hippocampus of a transgenic mouse model of AlzheimerГÇÖs disease. Mol Neurodegener 8:8-8

Fekete A, Vizi ES, Kovacs KJ, Lendvai B, Zelles T (2008) Layerspecific differences in reactive oxygen species levels after oxygen-glucose deprivation in acute hippocampal slices. Free Radic Biol Med 44(6):1010-1022

Forster MJ, Dubey A, Dawson KM, Stutts WA, Lal H, Sohal RS (1996) Age-related losses of cognitive function and motor skills in mice are associated with oxidative protein damage in the brain. Proc Natl Acad Sci U S A 93(10):4765-4769

Freeman R, Hill HDW (1971) Fourier transform study of NMR spin-lattice relaxation of "progressive saturation". J Chem Phys 54:3367-3377

Frisoni GB, Ganzola R, Canu E, Rub U, Pizzini FB, Alessandrini F, Zoccatelli G, Beltramello A, Caltagirone C, Thompson PM (2008) Mapping local hippocampal changes in Alzheimer's disease and normal ageing with MRI at 3 Tesla. Brain 131(Pt 12):3266-3276

Fukui KOJ, Omoi NO, Hayasaka TAKA, Shinnkai TADA, Suzuki SHOZ, Abe KOUI, Urano SHIR (2002) Cognitive impairment 
of rats caused by oxidative stress and aging, and its prevention by vitamin E. Ann N Y Acad Sci 959(1):275-284

Gomes M, Negrato C (2014) Alpha-lipoic acid as a pleiotropic compound with potential therapeutic use in diabetes and other chronic diseases. Diabetol Metab Syndr 6(1):80

Gulyas AI, Buzsaki G, Freund TF, Hirase H (2006) Populations of hippocampal inhibitory neurons express different levels of cytochrome c. Eur J Neurosci 23(10):2581-2594

Haacke EM, Brown RW, Thompson MR, Venkatesan R (1999) Magnetic resonance imaging: physical principles and sequence design. Wiley

Hall DJ, Han SH, Chepetan A, Inui EG, Rogers M, Dugan LL (2012) Dynamic optical imaging of metabolic and NADPH oxidasederived superoxide in live mouse brain using fluorescence lifetime unmixing. J Cereb Blood Flow Metab 32(1):23-32

Han BH, Zhou Ml, Johnson AW, Singh I, Liao F, Vellimana AK, Nelson JW, Milner E, Cirrito JR, Basak J, Yoo M, Dietrich HH, Holtzman DM, Zipfel GJ (2015) Contribution of reactive oxygen species to cerebral amyloid angiopathy, vasomotor dysfunction, and microhemorrhage in aged Tg2576 mice. Proc Natl Acad Sci U S A 112(8):E881-E890

Hara K, Harris RA (2002) The anesthetic mechanism of urethane: the effects on neurotransmitter-gated ion channels. Anesth Analg 94(2):313-318 table of contents

Haxaire C, Turpin FR, Potier B, Kervern M, Sinet PM, Barbanel G, Mothet JP, Dutar P, Billard JM (2012) Reversal of agerelated oxidative stress prevents hippocampal synaptic plasticity deficits by protecting D-serine-dependent NMDA receptor activation. Aging Cell 11(2):336-344

Hirata N, Shim YH, Pravdic D, Lohr NL, Pratt PF Jr, Weihrauch D, Kersten JR, Warltier DC, Bosnjak ZJ, Bienengraeber M (2011) Isoflurane differentially modulates mitochondrial reactive oxygen species production via forward versus reverse electron transport flow: implications for preconditioning. Anesthesiology 115(3):531-540

Hou C, Hsieh CJ, Li S, Lee H, Graham TJ, Xu K, Weng CC, Doot RK, Chu W, Chakraborty SK, Dugan LL, Mintun MA, Mach RH (2018) Development of a positron emission tomography radiotracer for imaging elevated levels of superoxide in neuroinflammation. ACS Chem Neurosci 9(3):578-586

Hsu JJ, Glover GH, Zaharchuk G (2009) Optimizing saturationrecovery measurements of the longitudinal relaxation rate under time constraints. Magn Reson Med 62(5):1202-1210

Huang W, Dong Y, Zhao G, Wang Y, Jiang J, Zhao P (2018) Influence of isoflurane exposure in pregnant rats on the learning and memory of offsprings. BMC Anesthesiol 18(1):5

Kadish I, Thibault O, Blalock EM, Chen K-C, Gant JC, Porter NM, Landfield PW (2009) Hippocampal and cognitive aging across the lifespan: a bioenergetic shift precedes and increased cholesterol trafficking parallels memory impairment. J Neurosci 29(6):1805-1816

Kanamaru T, Kamimura N, Yokota T, Iuchi K, Nishimaki K, Takami S, Akashiba H, Shitaka Y, Katsura Ki, Kimura K, Ohta S (2015) Oxidative stress accelerates amyloid deposition and memory impairment in a double-transgenic mouse model of Alzheimer's disease. Neurosci Lett 587:126-131

Kann O (2016) The interneuron energy hypothesis: implications for brain disease. Neurobiol Dis 90:75-85

Kehl F, Shen H, Moreno C, Farber NE, Roman RJ, Kampine JP, Hudetz AG (2002) Isoflurane-induced cerebral hyperemia is partially mediated by nitric oxide and epoxyeicosatrienoic acids in mice in vivo. Anesthesiology 97(6):1528-1533

Kubota R, Yamada S, Kubota K, Ishiwata K, Ido T (1993) Microautoradiographic method to study $[18 \mathrm{~F}] \mathrm{FDG}$ uptake in mouse tissue. Nucl Med Biol 20(2):183-188

Kugler P, Vogel S, Gehm M (1988) Quantitative succinate dehydrogenase histochemistry in the hippocampus of aged rats. Histochemistry 88(3-6):299-307

La C, Linortner P, Bernstein JD, Ua Cruadhlaoich MAI, Fenesy M, Deutsch GK, Rutt BK, Tian L, Wagner AD, Zeineh M, Kerchner GA, Poston KL (2019) Hippocampal CA1 subfield predicts episodic memory impairment in Parkinson's disease. Neuroimage Clin 23:101824-101824

Lacoste MG, Ponce IT, Golini RL, Delgado SM, Anzulovich AC (2017) Aging modifies daily variation of antioxidant enzymes and oxidative status in the hippocampus. Exp Gerontol 88:42-50

Li B, Feng XJ, Hu XY, Chen YP, Sha JC, Zhang HY, Fan HG (2018) Effect of melatonin on attenuating the isofluraneinduced oxidative damage is related to PKCalpha/Nrf2 signaling pathway in developing rats. Brain Res Bull 143:9-18

Lin D, Zuo Z (2011) Isoflurane induces hippocampal cell injury and cognitive impairments in adult rats. Neuropharmacology 61(8):1354-1359

Lowing JL, Susick LL, Caruso JP, Provenzano AM, Raghupathi R, Conti AC (2014) Experimental traumatic brain injury alters ethanol consumption and sensitivity. J Neurotrauma 31(20): 1700-1710

Ludwig LM, Tanaka K, Eells JT, Weihrauch D, Pagel PS, Kersten JR, Warltier DC (2004) Preconditioning by isoflurane is mediated by reactive oxygen species generated from mitochondrial electron transport chain complex III. Anesth Analg 99(5):1308-1315 table of contents

Mattiasson G, Friberg H, Hansson M, Elmer E, Wieloch T (2003) Flow cytometric analysis of mitochondria from CA1 and CA3 regions of rat hippocampus reveals differences in permeability transition pore activation. J Neurochem 87(2):532-544

McManus MJ, Murphy MP, Franklin JL (2011) The mitochondriatargeted antioxidant MitoQ prevents loss of spatial memory retention and early neuropathology in a transgenic mouse model of Alzheimer's disease. J Neurosci 31(44):15703-15715

Michalski R, Michalowski B, Sikora A, Zielonka J, Kalyanaraman B (2014) On the use of fluorescence lifetime imaging and dihydroethidium to detect superoxide in intact animals and ex vivo tissues: a reassessment. Free Radic Biol Med 67:278-284

Moser E, Moser M, Andersen P (1993) Spatial learning impairment parallels the magnitude of dorsal hippocampal lesions, but is hardly present following ventral lesions. J Neurosci 13(9):3916-3925

Mueller SG, Stables L, Du AT, Schuff N, Truran D, Cashdollar N, Weiner MW (2007) Measurement of hippocampal subfields and age-related changes with high resolution MRI at 4T. Neurobiol Aging 28(5):719-726

Navarro A, López-Cepero JM, Bández MJ, Sánchez-Pino M-J, Gómez C, Cadenas E, Boveris A (2008) Hippocampal mitochondrial dysfunction in rat aging. Am J Phys Regul Integr Comp Phys 294(2):R501-R509

Ni C, Li C, Dong Y, Guo X, Zhang Y, Xie Z (2017) Anesthetic isoflurane induces DNA damage through oxidative stress and p53 pathway. Mol Neurobiol 54(5):3591-3605 
Nicolle MM, Gonzalez J, Sugaya K, Baskerville KA, Bryan D, Lund K, Gallagher M, McKinney M (2001) Signatures of hippocampal oxidative stress in aged spatial learningimpaired rodents. Neuroscience 107(3):415-431

Paasonen J, Stenroos P, Salo RA, Kiviniemi V, Grohn O (2018) Functional connectivity under six anesthesia protocols and the awake condition in rat brain. Neuroimage 172:9-20

Pan W-J, Billings JCW, Grooms JK, Shakil S, Keilholz SD (2015) Considerations for resting state functional MRI and functional connectivity studies in rodents. Front Neurosci 9:269-269

Pipe JG (1999) Motion correction with PROPELLER MRI: application to head motion and free-breathing cardiac imaging. Magn Reson Med 42(5):963-969

Pratico D, Uryu K, Leight S, Trojanoswki JQ, Lee VM (2001) Increased lipid peroxidation precedes amyloid plaque formation in an animal model of Alzheimer amyloidosis. $\mathrm{J}$ Neurosci 21(12):4183-4187

Quick KL, Ali SS, Arch R, Xiong C, Wozniak D, Dugan LL (2008) A carboxyfullerene SOD mimetic improves cognition and extends the lifespan of mice. Neurobiol Aging 29(1):117-128

Raghavendra V, Kulkarni SK (2001) Possible antioxidant mechanism in melatonin reversal of aging and chronic ethanolinduced amnesia in plus-maze and passive avoidance memory tasks. Free Radic Biol Med 30(6):595-602

Raz N, Daugherty AM (2018) Pathways to brain aging and their modifiers: free-radical-induced energetic and neural decline in senescence (FRIENDS) model-a mini-review. Gerontology 64(1):49-57

Raz N, Daugherty AM, Bender AR, Dahle CL, Land S (2015) Volume of the hippocampal subfields in healthy adults: differential associations with age and a pro-inflammatory genetic variant. Brain Struct Funct 220(5):2663-2674

Safavynia SA, Goldstein PA (2019) The role of neuroinflammation in postoperative cognitive dysfunction: moving from hypothesis to treatment. Front Psychiatry 9:752-752

Santini E, Turner KL, Ramaraj AB, Murphy MP, Klann E, Kaphzan H (2015) Mitochondrial superoxide contributes to hippocampal synaptic dysfunction and memory deficits in Angelman syndrome model mice. J Neurosci 35(49):16213-16220

Schneider CA, Rasband WS, Eliceiri KW (2012) NIH image to ImageJ: 25 years of image analysis. Nat Methods 9(7):671-675

Schroeter A, Schlegel F, Seuwen A, Grandjean J, Rudin M (2014) Specificity of stimulus-evoked fMRI responses in the mouse: the influence of systemic physiological changes associated with innocuous stimulation under four different anesthetics. Neuroimage 94:372-384

Shetty RA, Ikonne US, Forster MJ, Sumien N (2014) Coenzyme Q10 and $\alpha$-tocopherol reversed age-associated functional impairments in mice. Exp Gerontol 58:208-218

Shimada M, Shimono R, Ozaki HS (1989) Freeze-mount microautoradiographic study in the mouse hippocampus after intravenous injection of tritiated 2-deoxyglucose and glucose. Neuroscience 31(2):347-354

Shimada M, Akagi N, Goto H, Watanabe H, Nakanishi M, Hirose Y, Watanabe M (1992) Microvessel and astroglial cell densities in the mouse hippocampus. J Anat 180(Pt 1):89-95

Shimada M, Kawamoto S, Hirose Y, Nakanishi M, Watanabe H, Watanabe M (1994) Regional differences in glucose transport in the mouse hippocampus. Histochem J 26(3):207-212
Stebbings KA, Choi HW, Ravindra A, Llano DA (2016) The impact of aging, hearing loss, and body weight on mouse hippocampal redox state, measured in brain slices using fluorescence imaging. Neurobiol Aging 42:101-109

Stoll S, Rostock A, Bartsch R, Korn E, Meichelbock A, Muller WE (1994) The potent free radical scavenger alpha-lipoic acid improves cognition in rodents. Ann N Y Acad Sci 717:122-128

Strange BA, Witter MP, Lein ES, Moser EI (2014) Functional organization of the hippocampal longitudinal axis. Nat Rev Neurosci 15(10):655-669

Toyama H, Ichise M, Liow JS, Vines DC, Seneca NM, Modell KJ, Seidel J, Green MV, Innis RB (2004) Evaluation of anesthesia effects on [18F]FDG uptake in mouse brain and heart using small animal PET. Nucl Med Biol 31(2):251-256

Tucsek Z, Toth P, Sosnowska D, Gautam T, Mitschelen M, Koller A, Szalai G, Sonntag WE, Ungvari Z, Csiszar A (2014) Obesity in aging exacerbates blood-brain barrier disruption, neuroinflammation, and oxidative stress in the mouse hippocampus: effects on expression of genes involved in betaamyloid generation and Alzheimer's disease. J Gerontol A Biol Sci Med Sci 69(10):1212-1226

Wang X, Pal R, Chen X-w, Limpeanchob N, Kumar KN, Michaelis EK (2005) High intrinsic oxidative stress may underlie selective vulnerability of the hippocampal CA1 region. Mol Brain Res 140(1):120-126

Wen Y, Li W, Poteet EC, Xie L, Tan C, Yan LJ, Ju X, Liu R, Qian H, Marvin MA, Goldberg MS, She H, Mao Z, Simpkins JW, Yang SH (2011) Alternative mitochondrial electron transfer as a novel strategy for neuroprotection. J Biol Chem 286(18): 16504-16515

Wilde GJC, Pringle AK, Wright P, Iannotti F (1997) Differential vulnerability of the CA1 and CA3 subfields of the hippocampus to superoxide and hydroxyl radicals in vitro. $\mathrm{J}$ Neurochem 69(2):883-886

Wu J, Li H, Sun X, Zhang H, Hao S, Ji M, Yang J, Li K (2015) A mitochondrion-targeted antioxidant ameliorates isofluraneinduced cognitive deficits in aging mice. PLoS One 10(9): e0138256

Xiao Y, Meierhofer D (2019) Are Hydroethidine-based probes reliable for reactive oxygen species detection? Antioxid Redox Signal 31(4):359-367

Xu F, Qiao S, Li H, Deng Y, Wang C, An J (2018) The effect of mitochondrial complex I-linked respiration by isoflurane is independent of mitochondrial nitric oxide production. Cardiorenal Med 8(2):113-122

Zhang J, Gao J, Guo G, Li S, Zhan G, Xie Z, Yang C, Luo A (2018) Anesthesia and surgery induce delirium-like behavior in susceptible mice: the role of oxidative stress. Am J Transl Res 10(8):2435-2444

Zimin PI, Woods CB, Kayser EB, Ramirez JM, Morgan PG, Sedensky MM (2018) Isoflurane disrupts excitatory neurotransmitter dynamics via inhibition of mitochondrial complex I. Br J Anaesth 120(5):1019-1032

Publisher's note Springer Nature remains neutral with regard to jurisdictional claims in published maps and institutional affiliations. 